\title{
The Perioperative Cardiac Risk Assessment: Medical and Legal Details Beyond the Cardiac Risk Calculator
}

\author{
Timothy E Paterick* \\ Aurora Health Care, Milwaukee/Green Bay, USA
}

*Corresponding author: Timothy E Paterick, Aurora Health Care, Milwaukee/Green Bay, USA.

Received Date: November 07, 2019

Published Date: November 13, 2019

\begin{abstract}
Cardiologists performing preoperative cardiac evaluations for non-cardiac surgery have a unique opportunity to assess and optimize the patient's baseline and general health; determine the patient's inherent surgical risk based upon a comprehensive history, physical examination and pertinent laboratory data; ensure the patient has made an informed choice regarding surgery, and identify post-operative risks that must be considered to reduce the potential for major adverse cardiovascular events. The introduction of perioperative risk calculators may have unintentionally reduced perioperative cardiac risk assessment into a singular thought process based upon a number generated by statistical analysis and computergenerated digit attempting to quantify risk. The displacement of the comprehensive clinical history and physical examination by an algorhythm may have negatively impacted our true risk assessment of patients undergoing surgery. The comprehensive subjective and objective evaluation allows appropriate decision-making regarding the proposed surgery and should allow accurate assessment of risks and have potential to reduce the patient's cardiovascular risk. Additionally, there is always a small inherent risk in all surgical procedures and operations. When an adverse outcome occurs, there is potential for an allegation of negligence. Any allegation of negligence will result in a detailed autopsy of the medical record. The best defense to an allegation of negligence is a true physician-patient relationship and comprehensive documentation in the medical record of the patient's assessment, clinical conditions impacting decision-making, and a detailed rational for the cardiologist's management decisions.
\end{abstract}

\section{Introduction}

Preoperative assessment of patients prior to noncardiac surgery is a common issue confronted by cardiologists. The field of perioperative medicine has been besieged with multiple risk calculators for cardiac assessment [1,2]. The risk calculators have value in detecting risk factors for major cardiovascular outcomes. The calculators are easy to use and often separate patients into high risk and low risk subsets. The original cardiac risk index [3] involved prospective clinician input and the bedside history and physical examination with amalgamation of all pertinent data points to determine the degree of surgical risk. The newer tools (risk calculators) have relied on probability and statistical models based upon retrospective medical data. Many important clinical variables may not have been included in the risks calculators. Patient may go through the fragmented US health care system without recognition of important clinical diagnoses such as: sleep apnea, pulmonary hypertension, diastolic heart failure, chirrhosis, and chronic obstructive pulmonary disease. Althought these patients manage their daily routines, these occult illnesses may have unintended consequence on patients in the perioperative period. Reliance on risk calculators may allow for important clinical variables such as dyspnea on exertion, irregular heart rhythms, aortic stenosis, alcohol abuse, and cognitive impairment to be missed. This makes it evident that an exhaustive and systematic clinical history and physical examination remain the cornerstone of the physician preparing to care for the perioperative patient. This preparation allows for the prevention and management of clinical decompensation. State of the art perioperative medical and surgical care is best accomplished through the combination of the science and art of medicine that involves a comprehensive history, physical examination, and review of lab and imaging data. The risk calculator is just an adjuvant decision aid and not a replacement for a true clinical assessment in real time. Since 1996, the American College of Cardiology/American Heart Association (ACC/AHA) has published four guideline documents addressing this issue, most recently in 2014 [4]. The goal of the clinician is to quantify the risk of surgery, which can help optimize the timing of the surgery, and manage the cardiac risk to hopefully reduce the morbidity and mortality of the procedure. However, less clear is the legal responsibility the clinician takes on, and what should be included 
in the documentation for a patient sent for "cardiac clearance". Herein we review the medical and explore the legal aspects of the preoperative cardiac evaluation.

\section{Cardiac Morbidity and Mortality for Non-Cardiac Surgery}

There are three major parameters to help determine the risk of cardiac morbidity and mortality for patients with whom noncardiac surgery is planned: (1) the urgency of the procedure, (2) the inherent cardiac risk of the planned surgical procedure, and (3) the patient's clinical markers assessing risk and functional status. The clinician should then integrate and synthesize this information to develop an estimate of perioperative cardiac risk and determine the need for additional testing or specific pharmacologic therapy prior to the surgery [5].

\section{Medical Necessity and Timing of Non-Cardiac Surgery}

For any procedure, consideration of its medical necessity and timing are important. Procedures are categorized as emergent, urgent, time-sensitive, and elective. An emergent procedure is one in which life or limb is threatened if the patient is not taken to the operating room within six hours. An urgent procedure is one in which life or limb is threatened if the patient does not have the procedure between 6 and 24 hours. A time-sensitive procedure means a delay of greater than one to six weeks will negatively affect outcome. Finally, an elective procedure is one that could be delayed up to one year. Non-cardiac surgery should proceed as soon as possible for emergent procedures without extensive preoperative cardiac assessment, as such testing could delay potentially lifesaving surgery $[4,5]$.

\section{The Proposed Surgery - Specific Inherent Risk}

The cardiologist should consider the surgery-specific risk; the inherent risk associated with the procedure. The ACC/AHA criteria for a low risk procedure is one that has a cardiovascular risk of $<1 \%$. Procedures that fall into this category include: endoscopic, ophthalmologic, dermatologic, and breast surgical procedures. A procedure with a cardiovascular risk $>1 \%$ is identified as having an elevated risk. The ACC/AHA no longer classifies risk as low, intermediate or high because recommendations for the latter two are the identical. The ESC continues to use low, intermediate, and high risk, associated with cardiovascular risks of $<1 \%, 1-5 \%$, and $>5 \%$ respectively [4-6].

\section{Clinical Predictors and Physical Examination Findings}

Recommendations regarding surgical risk and management are largely based upon the clinical predictors identified in patient's history and physical examination. During the preoperative evaluation cardiologists would be prudent to inquire about symptoms such as: angina, dyspnea, syncope, and palpitations. Additionally, any past history of cardiac, cerebrovascular, peripheral arterial disease, hypertension, diabetes, and chronic kidney disease are important in risk assessment. The physical examination should focus on the cardiovascular system, and include blood pressure measurements, auscultation of the heart and lungs, abdominal palpation, and examination of the extremities for edema and vascular integrity. Important findings include carotid artery bruits, evidence of heart failure, a murmur suspicious for hemodynamically significant valvular heart disease, pulmonary hypertension, and evidence of significant peripheral vascular disease.

\section{Cardiac Conditions that Elevate Risk}

The presence of certain cardiac conditions greatly increase the risk of cardiac morbidity and mortality, and are referred to by the ACC/AHA guidelines as active cardiac conditions including: (1) acute ( $\leq 7$ days), or recent ( $>7$ but $\leq 30$ days) myocardial infarction, or unstable angina identified as Canadian cardiovascular society class 3 or 4, (2) decompensated heart failure, (3) severe valvular lesions, especially aortic and mitral stenosis, and (4) significant arrhythmias, including high grade atrioventricular block and ventricular arrhythmias [4,5]. These active conditions preclude proceeding with noncardiac surgery without further evaluation and management, unless emergent noncardiac surgery is indicated.

\section{Other Cardiac Risk Factors Associated with Increased Risk}

If there are no active cardiac conditions to preclude surgery the clinician should investigate clinical risk factors that have been associated with an increased risk of cardiac events for noncardiac surgery. The clinical cardiac risk factors used in the ACC/ AHA guidelines are derived from the Revised Cardiac Risk Index, described by Lee et al. which uses a six-point index score for assessing the risk of complications with non-cardiac surgery [7]. The Revised Cardiac Risk Index (RCRI) includes the following variables and risks:

a. Ischemic heart disease

b. Congestive heart failure

c. History of cerebrovascular disease

d. Diabetes requiring insulin therapy

e. Preoperative serum creatinine level higher than $2 \mathrm{mg} / \mathrm{dL}$

f. High-risk surgery (intrathoracic, intraabdominal. or supraliminal vascular)

Each of the six risk factors is assigned one point. Patients with 0 1,2 , and $>3$ risk factors are associated with $0.4 \%, 1 \%, 2.4 \%$, and $5.4 \%$ of adverse cardiac events. This index is simple, has been extensively validated, and provides a good estimate of the preoperative risk. Patients with 0-1 clinical risk factors are low risk and require no additional cardiovascular testing. Patients with $\geq 2$ risk factors are higher risk and may require additional cardiovascular evaluation depending on their functional capacity [7].

\section{Functional Capacity}

Determining a patient's functional capacity is vital, as exercise capacity is a reliable predictor of future cardiac events. It is usually 
expressed in metabolic equivalents (METs), where one MET is defined as the oxygen consumption of a $70 \mathrm{-kg}$ man at rest. A functional capacity of 4 METS is generally the cutoff for acceptable functional capacity to undergo surgery because it is typically equivalent to the physiologic stress of most noncardiac surgical procedures requiring general anesthesia. The Duke Activity Status Index suggests questions that correlate with MET levels; for example, walking on level ground at about 4 miles per hour, or walking up a flight of stairs expends approximately 4 METs of activity. In patients who can perform $\geq 4$ METs of activity the recommendations suggest no additional testing is needed. Patients whose functional capacity is lower or cannot be assessed due to limitations in their activity level may benefit from additional cardiac testing [5].

\section{Supplemental Preoperative Evaluation}

The above clinical and examination information should be integrated to recommend proceeding with surgery or contemplate whether the patient would benefit from further evaluation and testing. Supplemental preoperative cardiac evaluation may include an ECG, assessment of LV function, and exercise or pharmacological stress testing $[4,5]$.

\section{A. $\quad$ ECG}

i. $\quad$ No benefit (Class 3) of ECG for asymptomatic patients undergoing low risk surgery

ii. For patients undergoing elevated risk surgery: ECG is reasonable (Class 2a) in patients with known CAD, significant arrhythmia, PVD, CVD, or other significant structural heart disease, and may be considered (Class $2 b)$ in asymptomatic patients.

B. Assessment of LV function

i. Reasonable (Class 2a) in patients with dyspnea of unknown origin, or patients with a history of heart failure with a change in clinical status

C. Stress testing

i. Stress testing is not indicated in 2 circumstances: (1) patients at low risk for noncardiac surgery (Class 3), (2) patients with elevated risk and excellent ( $>10$ Mets) functional capacity (Class 2a), or moderate to good (4-10 Mets) functional capacity (Class $2 \mathrm{~b}$ )

ii. Stress testing with dobutamine stress test or myocardial perfusion imaging is reasonable (Class 2a) for patients with poor or unknown functional capacity. Exercise stress testing may also be considered (Class 2b) for patients with poor or unknown functional capacity. In either case stress testing should be performed only if the results would change management

D. Cardiologists performing consultations for non-cardiac surgery would be prudent to include the following information in the medical record to optimize patient care and minimize the potential for allegations of negligence
I. The proposed surgical procedure and the surgery specific risks and benefits

II. The patient's understanding of the benefits and risks of the surgical procedure

III. The presence or absence of any active cardiac conditions including angina, heart failure, valve disease, arrhythmias and claudication

IV. The presence or absence of cardiac risk factors including any history of ischemic heart disease, heart failure, cerebrovascular disease, diabetes mellitus requiring insulin therapy, and a serum creatinine greater than $2 \mathrm{mg} / \mathrm{dL}$

V. The patient's functional capacity with a detailed description of the exercise capacity and whether it exceeds the 4 Mets that is typically identified as equivalent to the physiologic stress of most noncardiac surgical procedures

\section{Discussion}

Medical and legal considerations for clinicians performing pre-op evaluations for cardiac clearance of non-cardiac surgery are imperative. Pre-op risk assessment for cardiac clearance for non- cardiac surgery is frequently complex, and like any predictive process is imperfect and saddled with a degree of uncertainty. A small percent of patients will experience an untoward, adverse clinical event despite comprehensive work-up and evaluation. There is small, but inherent risk, in all surgical procedures and operations. When an adverse outcome occurs, there is potential for an allegation of negligence. The allegation of negligence will result in a detailed autopsy of the medical record. The best defense to an allegation of negligence is comprehensive documentation in the physician's note in the medical record of the patient's assessment and clinical conditions. Also, it is imperative to delineate the clinical rationale for the decision-making, and there must be clear documentation of the potential risks associated with the noncardiac surgery.

The medical record should outline succinctly and concisely the descriptive clinical features of the patient's disease processes and how they are impacting the patient's health and well-being, the known inherent cardiac risk of the planned surgical procedure, and a description of the patient's functional capacity - outlining whether they meet the time honored activity level of 4 METS requirement, which is felt to be the equivalent to the physiologic stress of most non-cardiac procedures. Additionally the medical record documentation should reflect a review of the six clinical risk factors impacting decisions regarding the use of medical therapy or the need for further evaluation for cardiac disease, Specifically the medical record should reflect the presence or absence of: 1) ischemic heart disease, 2) heart failure, 3) cerebrovascular disease, 4) diabetes, 5) renal dysfunction and 6) the level of risk associated with the planned surgery. The medical record note should also outline any therapeutic consideration necessary pre- 
and post-operatively with beta-blockers to control heart to less than 65 beat per minute and the use of statin therapy. The need for constant surveillance of blood pressure, heart rate, cardiovascular status post operatively should be clearly defined in the medical record. Ultimately a true physician - patient partnership and the medical record are the best defenses against any allegations of negligence. If there is no documentation in the electronic medical record, it will be assumed that the proper evaluation did not occur, even if it did occur. Physicians performing preoperative cardiac evaluations ideally should perform a comprehensive assessment as outlined above to identify the optimal approach to patient care and management and should detail in the medical record the detailed assessment that supports the clinical recommendations. This documentation will likely reduce the physician's risk for allegations of negligence [8].

\section{Acknowledgements}

None.

\section{Conflict of Interest}

No conflict of interest.

\section{References}

1. Bilimoria KY, Liu Y, Paruch JL, Zhou L, Kmiecik TE, et al. (2013) Development and evaluation of the universal ACS NSQIP surgical risk calculator: a decision aid and informed consent tool for patients and surgeons. J Am Coll Surg 215(5): 833-842.
2. Gupta PK, Gupta H, Sundaram A, Kaushik M, Fang X, et al. (2011) Development and validation of a risk calculator for prediction of cardiac risk after surgery. Circulation 124(4): 381-387.

3. Goldman L, Caldera DL, Nussbaum SR, Southwick FS, Krogstad D, et al. (1977) Multifactorial index of cardiac risk in noncardiac surgical procedures. NEJM 297(16): 845-850.

4. Fleisher LA, Fleischmann KE, Auerbach AD, Barnason SA, Beckman JA, et al. (2014) ACC/AHA Guideline on Perioperative Cardiovascular Evaluation and Management of Patients Undergoing Non-cardiac Surgery: Executive Summary, Journal of the American College of Cardiology. Circulation 130(24): 2215-2245.

5. William K Freeman, Raymond J (2009) Gibbons MD Perioperative Cardiovascular Assessment of Patients Undergoing Non-cardiac Surgery Mayo Clinic Proceedings 84(1): 79-90.

6. Fleisher LA, Beckman JA, Brown KA, Calkins H, Chaikof E, et al. (2007) ACC/AHA 2007 guidelines on perioperative cardiovascular evaluation and care for non-cardiac surgery: a report of the American College of Cardiology/American Heart Association task force on practice guidelines (writing committee to revise the 2002 guidelines on perioperative cardiovascular evaluation for non-cardiac surgery). Circulation 116: e418-e500.

7. Lee TH, Marcantonio ER, Mangione CM, Thomas EJ, Polanczyk CA, et al. (1999) Derivation and prospective validation of a simple index for prediction of cardiac risk of major non-cardiac surgery. Circulation 100: 1043-1049.

8. Paterick TJ, Carson GV, Allen MC, Paterick TE (2008) Medical Informed Consent: General Considerations for Physicians. Mayo Clinic Proc 83(3): 313-319. 